PSICOLOGÍA

IBEROAMERICANA
Psicología Iberoamericana ISSN: 1405-0943

revista.psicologia@ibero.mx

Universidad Iberoamericana, Ciudad de México México

\title{
Significado psicológico, roles y expectativas de la paternidad en adolescentes mexicanos: Estudio exploratorio
}

Balam Aguilar, Janer Manuel; Valdes Santiago, Ana Gabriel; Ramírez Marmolejo, Arturo; Padilla Gámez, Nélida

Significado psicológico, roles y expectativas de la paternidad en adolescentes mexicanos: Estudio exploratorio Psicología Iberoamericana, vol. 26, núm. 1, 2018

Universidad Iberoamericana, Ciudad de México, México

Disponible en: http://www.redalyc.org/articulo.oa?id=133959553006 


\section{Significado psicológico, roles y expectativas de la paternidad en adolescentes mexicanos: Estudio exploratorio}

Psychologial meaning, roles and expectations about paternity in Mexican adolescents: Exploratory study

Janer Manuel Balam Aguilar janer.baguilar@gmail.com Universidad Nacional Autónoma de México , México

Ana Gabriel Valdes Santiago

Universidad Nacional Autónoma de México, México

Arturo Ramírez Marmolejo

Universidad Nacional Autónoma de México, México

Nélida Padilla Gámez

Universidad Nacional Autónoma de México, México

Resumen: En el presente trabajo se describen el significado psicológico, roles y expectativas asociadas a la paternidad en hombres y mujeres adolescentes de la Ciudad de México, mediante la técnica de redes semánticas naturales modificadas. Los resultados muestran la permanencia del rol proveedor en el padre en ambos sexos; no obstante, existen diferencias entre sexos en términos de expectativas de la paternidad, sugiriendo un alejamiento de este rol hacia una imagen más involucrada en el cuidado, atención y afecto. Se vislumbra la resignificación de la paternidad para los jóvenes, lo cual conlleva a cuestionarse sobre la educación familiar, así como sobre los retos y oportunidades socioculturales de su fortalecimiento en los jóvenes, lo que podría ser el inicio de un cambio hacia las nuevas paternidades que se intentan ejercer en la actualidad.

Palabras clave: paternidad, adolescentes, género, familia, México.

Abstract: This paper describes the psychological meaning, roles and expectations associated to paternity in men and women adolescents from Mexico City, through the technique of Modified Natural Semantic Networks. The results show the permanence of the father as a provider in both sexes; nevertheless, there are differences between genders in terms of paternity expectations, which suggest distancing from this role towards an image more involved in caring, attention and affection. A resignification of paternity in the younger generations is foreseen, which carries to question family education, as well as the sociocultural challenges and opportunities for its strengthening among the youth, which could be the beginning of a change towards the new paternities being attempted to exercise nowadays.

Keywords: paternity, adolescents, gender, family, Mexico.

\section{INTRODUCCIÓN}

En la última década, el interés hacia el estudio de la paternidad ha incrementado de manera significativa debido a las transformaciones ideológicas que cuestionan el modelo tradicional de una familia contemporánea; esto ha generado divergencias en su estructura, funcionamiento e idiosincrasia cada vez más visibles dentro de la sociedad actual. 
Como señalan Mena y Torres (2013), en México, el estudio de la paternidad ha sido encauzado hacia ciertos fenómenos disidentes de la paternidad tradicional, con mayor atención a grupos poblacionales paternalmente activos, adultos o infantiles y, principalmente, de varones. Pocos son los estudios que han tomado a los jóvenes como protagonistas de este fenómeno, en particular, cuando éstos se encuentran en un proceso de aprendizaje sobre su parentalidad, así como a la invisibilización de la influencia femenina dentro de esta etapa.

La perspectiva de género toma un papel importante dentro de este fenómeno debido a la demostración teórica que enmarca la opresión de géneros como resultado del sistema de significados atribuidos a las actividades que realizan las personas en su interacción social concreta, y no su condición biológica. Esto dota al género de una carácter multiversal (Ramírez, 2006) que permite el cuestionamiento, la crítica y reflexión sobre las construcciones que se tienen sobre las múltiples imágenes, tareas, expectativas y expresiones que puede tener un varón dentro de un contexto sociocultural, posibilitando la exploración de sus roles paternos en términos económicos, de protección y cuidado, autoridad, manutención del hogar (Barker, Aguayo, Kimelman, \& Figueroa, 2011) y afectivos.

\section{Paternidad}

De acuerdo con la Comisión Económica para América Latina y el Caribe (Cepal, 2001), la paternidad se define como un fenómeno cultural, social y subjetivo que relaciona a los hombres con sus hijos e hijas, en el marco de una práctica compleja en la que intervienen diversos factores en constante transformación, trascendiendo de cualquier tipo de arreglo conyugal.

Salguero (2006) enmarca la paternidad como una relación social compleja, que trasciende del hecho de engendrar, al involucrar dimensiones como la provisión, autoridad, protección y transmisión de saberes; las cuales, de acuerdo con Minuchin y Fishman (1984) delimitan la herencia cultural del futuro en una relación bidireccional innegable.

De esta forma, las diversas sociedades han construido simbolismos acerca de las actividades propias de un padre, generalmente institucionalizadas y normadas por las expectativas socioculturales de género. En el caso particular de México, el hombre responde a diferentes premisas-histórico-socio-culturales (phsc) que regulan su acceso o restricción hacia escenarios, comportamientos y creencias del cómo ser padre dentro de un núcleo familiar (Díaz-Loving, Rivera, Villanueva, \& Cruz, 2011), a su vez, la mujer y su progenie se encuentran permeados de esta normativa que los hace arraigarse a los reglamentos socioculturales vigentes en su contexto.

Correa, García y Saldívar (2013) iluminan la fuerte asociación entre los conceptos del ser padre y el ser hombre, presentes en los varones adolescentes mexicanos. De este modo, al continuar prevaleciendo premisas relacionadas con los roles tradicionales de la mujer, del hombre y la supremacía masculina (Salvídar et al., 2015), es lógico pensar que 
estas ideologías imperen en el actuar del hombre en su ejercicio parental, tanto en sus hijos como en la relación con su pareja; sobre todo, cuando ésta podría tender a esperar o reforzar ciertos estereotipos de género masculinos.

Diversos son los estudios que asocian a un padre con el trabajo, la provisión económica, el liderazgo de la familia y la desprovista capacidad emocional en sus cuidados (Bonino, 2003; Correa et al., 2013; Castillo, 2015; Nieri, 2012). Esto, de acuerdo con Correa et al. hace inferir la fuerte correspondencia entre el concepto paterno, el ejercicio masculino y las actividades conferidas al mismo; lo que les hace concebirse con el derecho de ser obedecidos a costo de la provisión económica y la pérdida de la libertad tanto personal como sexual.

Respecto a este último punto, se identifica una ambivalencia en los varones ante los costos de los beneficios al ejercer el rol masculino (Correa et al., 2013). Esta observación puede encontrarse asociada debido a los cambios vislumbrados en algunas premisas culturales que abogan por la neutralidad en las tendencias de género (Saldívar et al., 2015), mismos que se asocian a los diversos cambios socioculturales existentes en la población, con énfasis en aquellos que se apartan de la cultura tradicional mexicana o, como los llamaría Díaz-Guerrero y Castillo-Vales (1981), contraculturales, que interceden por un cambio en los valores y premisas socioculturales que median las dinámicas entre seres humanos.

De este modo, si existe evidencia de ciertos cambios en los valores culturales de género de la población mexicana (Díaz-Loving et al., 2015; García-Campos, 2008; Saldívar et al., 2015), al igual que aquella que demuestra cambios conductuales en el ejercicio masculino de los hombres mexicanos (Barker et al., 2011), ¿existirá algún cambio en la construcción del significado de paternidad, así como en las actividades y expectativas del mismo? Los adolescentes, al ser un grupo en constante debate de los valores tradicionales y postmodernos, representan un grupo relevante, debido a que constituyen el reflejo actual de la construcción de identidades y de creencias sobre patrones de género (Cubillas et al., 2016) que se reproducen, valúan y critican en la sociedad; es por ello, que los consideramos como un grupo idóneo para explorar dicho constructo.

\section{Paternidad y Adolescencia}

De acuerdo con Brañas (1997), la adolescencia es un periodo transitorio comprendido entre los 10 y 21 años de edad; aunque, como señala Gaete (2015), es un proceso debatible debido al nulo consenso que existe entre los teóricos sobre su inicio y fin, sobre todo, cuando se han encontrado señales de un adelanto de la pubertad, la cual suele determinar el comienzo de la adolescencia, y un retraso hacia la adquisición de los hitos psicosociales que delimitan su finalización.

Este argumento retoma importancia en la contemplación de la paternidad como el cumplimiento de una expectativa en el rol de género masculino (Paterna, Martínez, \& Rodes, 2005), en la cual recaen nuevas precisiones de identidad y relación entre los varones, las cuales suelen 
reforzarse en la adolescencia (Connell, 2003) debido a la alta socialización entre el individuo y sus múltiples círculos sociales.

Asimismo, Aguirre y Guell (2002) indican que en la adolescencia ocurren los cambios de carácter más importantes del hombre, los cuales están influidos fuertemente por la cultura, a la par de factores fisiológicos y sociales que otorgan a cada varón diversos significados sobre su masculinidad y la expresión de la misma (Cultura Salud/Sename, 2011).

Tal cual lo señala un estudio realizado por Barker et al. (2011) en el que se destaca la importancia de la edad en el aprendizaje de las conductas equitativas de género, ya que aquellos que son más jóvenes presentan mayor apertura hacia actitudes género-equitativas $y$, por consiguiente, mayor expresión del mismo en escenarios sociales; la adolescencia representa una etapa crítica en el aprendizaje y práctica de actitudes psicosociales que nutrirán fundamentalmente su etapa adulta. De este modo, el presente estudio brinda un acercamiento a los significados que los adolescentes, tanto hombres como mujeres, asocian a la paternidad y, por consiguiente, a diferentes constructos que la preceden, como la masculinidad y la equidad de género, para así aproximarse al estudio de este fenómeno en un grupo poblacional relevante y abrir el diálogo y la reflexión hacia las necesidades que presenta dicho grupo; asimismo, producir un trabajo tanto teórico como práctico cercano a su percepción social, que produzca un impacto y trascendencia en los jóvenes, cuyas acciones, pensamientos, afectos y proyecciones del hoy, representarán las transformaciones socioculturales del mañana.

Continuando en esta línea, aproximarse al conocimiento del paralelismo entre los valores culturales adolescentes y los valores dirigidos hacia una equidad de género podría esclarecer diferentes alternativas que implican actitudes asociadas a la liberación sociocultural de las expectativas de género que reglamentan el desenvolvimiento personal, conllevando problemas de salud (Aguirre \& Guell, 2002) y malestares sociales (Bonino, 2003) que afectan el bienestar personal.

Por otra parte, conocer los significados que asocian a las mujeres con la paternidad devela una perspectiva complementaria y bidireccional en la función del varón como padre. Al respecto, Figueroa y Flores (2012) remarcan el papel de las construcciones de género, las cuales limitan la participación de los varones en actividades o contextos socialmente establecidos para la mujer; pues tanto varones como mujeres se sienten incómodos al transgredir espacios tradicionalmente asociados a otro género (Mena \& Torres, 2013) y, a su vez, las mujeres no permiten su involucramiento en actividades que consideran les pertenecen (Figueroa \& Flores, 2012), ya que, como señala Lara (2000, citado en Saldívar et al., 2015), la necesidad de ajuste social impera en las respuestas de hombres y mujeres en la sociedad.

$\mathrm{Al}$ respecto, la perspectiva de género resalta un enfoque relacional entre hombres y mujeres, en el cual lo socialmente establecido como femenino se encuentra en estrecho vínculo con lo socialmente establecido como masculino, y viceversa. De este modo, ambos sexos derivan en inequidades, privilegios y desventajas producidos por los 
mandatos socioculturales (Cultura Salud/Sename, 2011), por lo cual, su cuestionamiento, crítica y sucesiva deconstrucción ha de ser tanto por hombres como por mujeres, pues ambos ponen en juego el poder y tradición que en sus escenarios permean, incluyendo el parental.

De igual modo, el presente estudio pretende describir los indicadores cognitivos y conductuales del constructo en cuestión, con la intención de establecer un acercamiento en la población joven para la construcción de algún instrumento que mida las actitudes hacia las paternidades disidentes, en término de mayor consciencia de su rol, demostración de afecto en público, cuidados democráticos, emocionales y equitativos, así como crianza comprometida y emocionalmente satisfactoria para el hombre, y por consiguiente a la mujer.

Debido a que la paternidad, bajo el enfoque de género, es un fenómeno reciente y de una complejidad evidente, la técnica de redes semánticas naturales modificadas (Reyes-Lagunes, 1993) representa una técnica plausible para el abordaje de este fenómeno, debido a su eficiencia en obtener conductas e indicadores objetivos que determinarían la categorización de elementos o dimensiones que permitirían comprender, en término tangibles y cuantificables, la paternidad en la población juvenil mexicana.

Finalmente, este estudio manifiesta un panorama para las futuras investigaciones que tengan como objetivo abordar la paternidad desde una perspectiva de género, tanto en hombres como mujeres, ya que constribuye a la definición del fenómeno, la visibilización de experiencias y la instrumentalización de los mismos, para concretar una realidad psicosocial mexicana evidente, la cual necesita ser reconocida, valorada y empoderada.

\section{MÉTODO}

\section{Participantes}

La muestra, no probabilística y propositiva, estuvo conformada por 100 participantes, 50 hombres y 50 mujeres, con un rango de edad de entre 14 y 21 años $(M=16.96)$, estudiantes de nivel bachillerato (69\%) y licenciatura (31\%) de la zona oriente de la Ciudad de México.

\section{Técnica}

Redes semánticas naturales modificadas. Técnica de autoinforme semiestructurada propuesta originalmente por Figueroa, González y Solís (1981) y revisada por Reyes-Lagunes (1993), cuyo objetivo se centra en conocer el significado psicológico de uno o varios conceptos, así como identificar las conductas o indicadores que la población meta considere que pertenecen al constructo de interés.

Se emplearon cuatro estímulos totales, los cuales se diferenciaron de acuerdo con el sexo del participante. En el caso de los hombres, 
se aplicaron uno con la finalidad de conocer el significado psicológico ("Paternidad"), uno para determinar conductas ("Yo como padre"), uno para expectativas sociales ("Mi pareja esperaría que como padre yo") y un distractor ("Embarazo"). Por otra parte, en los estímulos para mujeres, se conservaron los referidos a significado psicológico y el distractor, pero se modificaron aquellos destinados a conductas ("Mi pareja como padre") y expectativas sociales ("Espero que mi pareja como padre"). La exposición de los estímulos fue acorde con el orden presentado, aleatorizando únicamente el estímulo distractor.

\section{Procedimiento}

La aplicación del instrumento dio inicio con el consentimiento informado de la participación y la lectura del aviso de confidencialidad y anonimato de la información proporcionada. Posteriormente, se aplicó la técnica de redes semánticas con los estímulos mencionados previamente. Finalmente, se analizaron cuantitativamente los datos recabados mediante una base de datos diseñada en Excel 2011, con lo que se obtuvo la siguiente información: tamaño de la red, peso semántico, núcleo de la red, distancia semántica cuantitativa e índice de consenso grupal.

\section{RESULTADOS}

\section{Significado Psicológico de La Paternidad}

De acuerdo con los datos obtenidos, el tamaño de la red fue similar tanto en hombres como en mujeres con 100 palabras definidoras del concepto, con un índice de consenso grupal de $60 \%$. A su vez, como se muestra en la Figura 1 y 2, el núcleo de la red, en ambos sexos, vincula el concepto de paternidad con términos como padre, responsabilidad, hijos, amor, cuidados y hombre; con la diferencia de que adicionalmente los hombres lo asocian con trabajo, padres y respeto; y las mujeres, con educación, dinero y protección. 


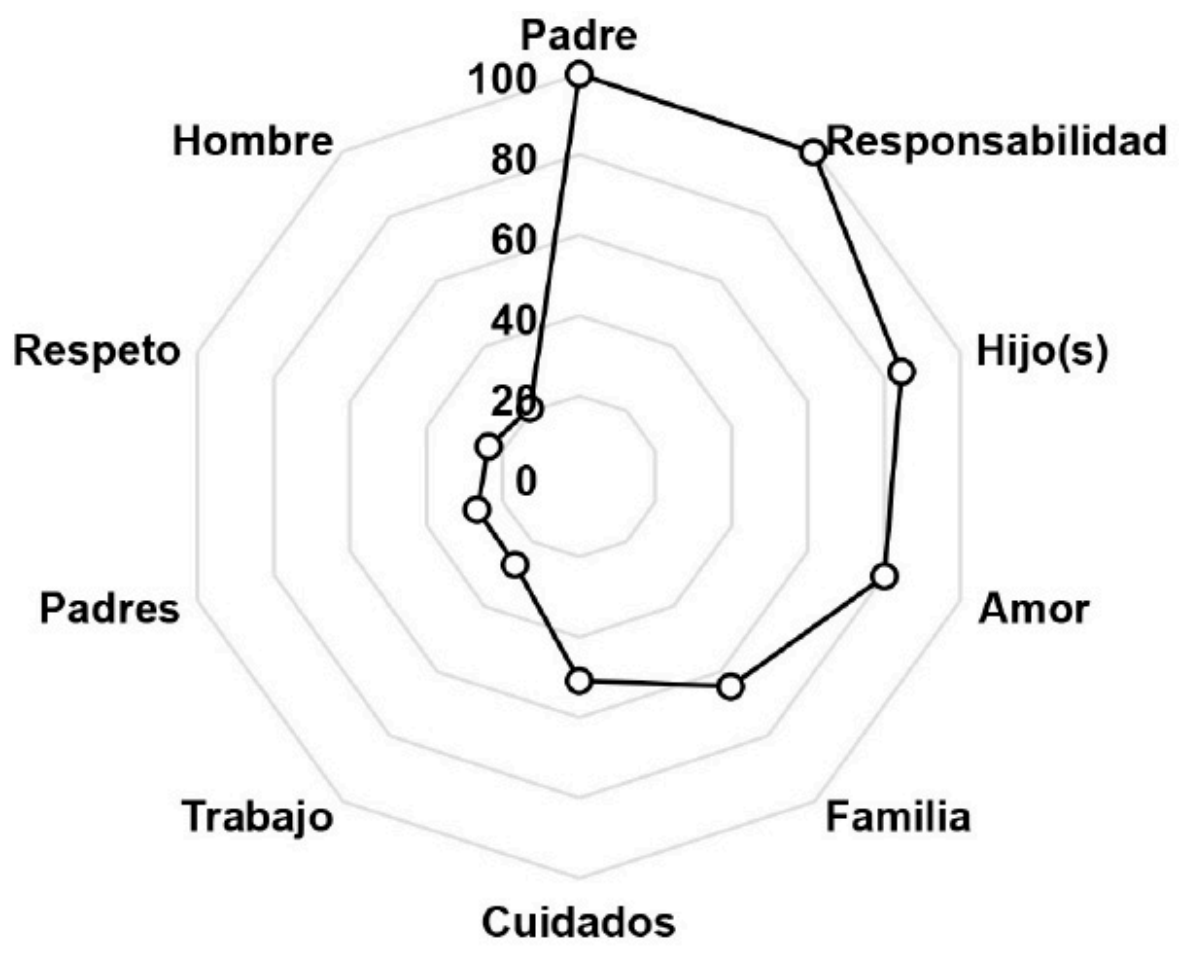

Figura 1

Núcleo de red de paternidad en hombres

Figura 1

Núcleo de red de paternidad en hombres 


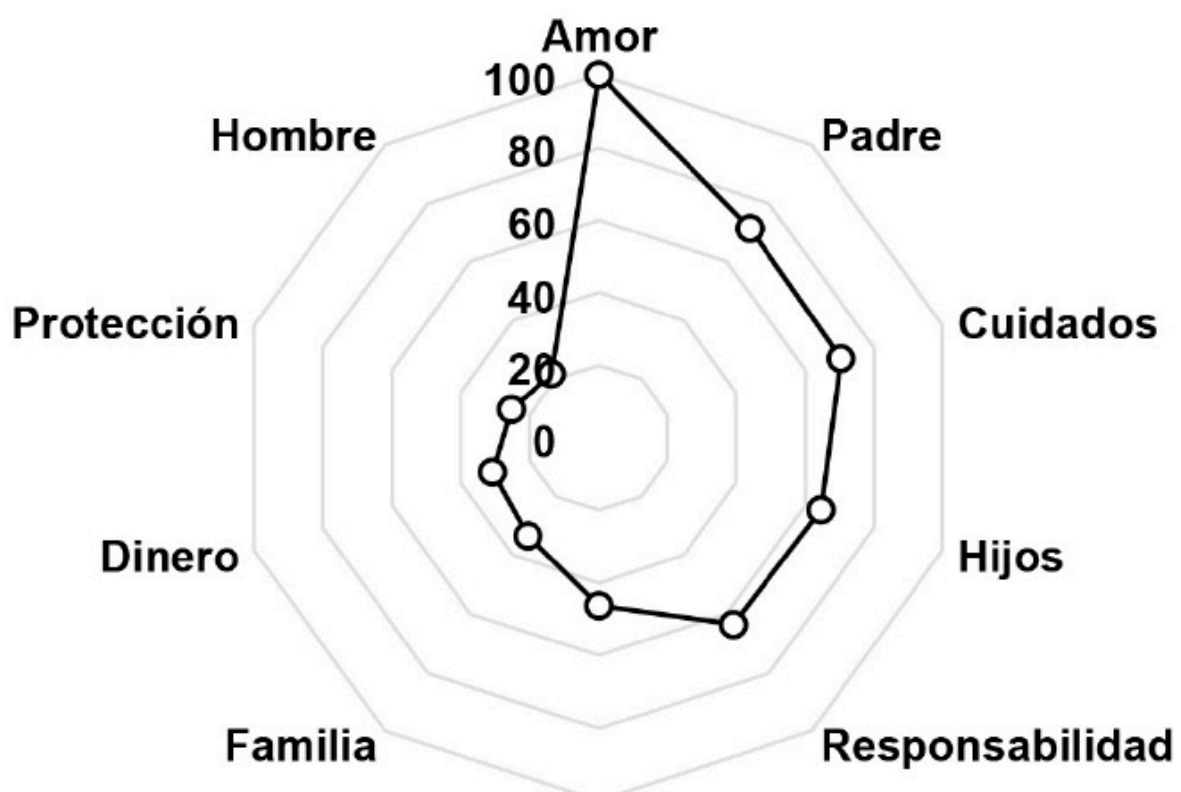

Educación

Figura 2

Núcleo de red de paternidad en mujeres

Figura 2

Núcleo de red de paternidad en mujeres

Nota. La distribución de valores del eje radial de las gráficas equivale a la Distancia Semántica Cuantitativa (dsc) de cada definidora del núcleo de red.

Asimismo, se señala la importancia del orden jerárquico entre las definidoras que componen los diferentes núcleos de red, dado que las similitudes entre éstas sematizan debido a los diferentes pesos semánticos y distancias semánticas cuantitativas, las cuales se presentan en la Tabla 1.

Tabla 1.

Núcleo de red, peso semántico y distancia semántica cuantitativa de paternidad en hombres y mujeres.

\begin{tabular}{|c|c|c|c|c|c|}
\hline \multicolumn{6}{|c|}{ Estímulo: Paternidad } \\
\hline \multicolumn{3}{|c|}{ Mujeres } & \multicolumn{3}{|c|}{ Hombres } \\
\hline Núcleo de red & ps & dsc & Núcleo de red & ps & dsc \\
\hline Amor & 301 & 100 & Padre & 206 & 100 \\
\hline Padre & 215 & 71.42 & Responsabilidad & 205 & 99.51 \\
\hline Cuidados & 212 & 70.43 & Hijo & 174 & 84.46 \\
\hline Hijos & 194 & 64.45 & Amor & 165 & 80.09 \\
\hline Responsabilidad & 191 & 63.45 & Familia & 133 & 64.56 \\
\hline Educación & 139 & 46.17 & Cuidados & 105 & 50.97 \\
\hline Familia & 100 & 33.22 & Trabajo & 56 & 27.18 \\
\hline Dinero & 92 & 30.56 & Padres & 55 & 26.69 \\
\hline Protección & 76 & 25.24 & Respeto & 49 & 23.78 \\
\hline Hombre & 66 & 21.92 & Hombre & 43 & 20.87 \\
\hline
\end{tabular}

Nota. Las siglas señaladas en la tabla corresponden a Peso semántico (ps) y Distancia semántica cuantitativa (dsc).

\section{Tabla 1}

Núcleo de red, peso semántico y distancia semántica cuantitativa de paternidad en hombres y mujeres Nota. Las siglas señaladas en la tabla corresponden a Peso semántico (ps) y Distancia semántica cuantitativa 


\section{Roles Relacionados con la Paternidad}

En cuanto al estímulo relacionado con los roles de la paternidad ("Yo como padre" / "Mi pareja como padre"), se identificaron tamaños de red ligeramente diferentes, entre las que destacan las mujeres con unas 118 palabras, a comparación de las 111 palabras emitidas por los hombres. Respecto al núcleo de red, tanto mujeres como hombres precisan que los padres tienen roles relacionados con la responsabilidad, amor, cuidados, comprensión, hijos, educación y familia, con la precisión de un índice de consenso grupal de 70\%. No obstante, los hombres dan énfasis al trabajo, el apoyo y el ser estricto, entretanto, las mujeres a desagradable, bondadoso y proveedor (Ver Figura 3 y 4 ).

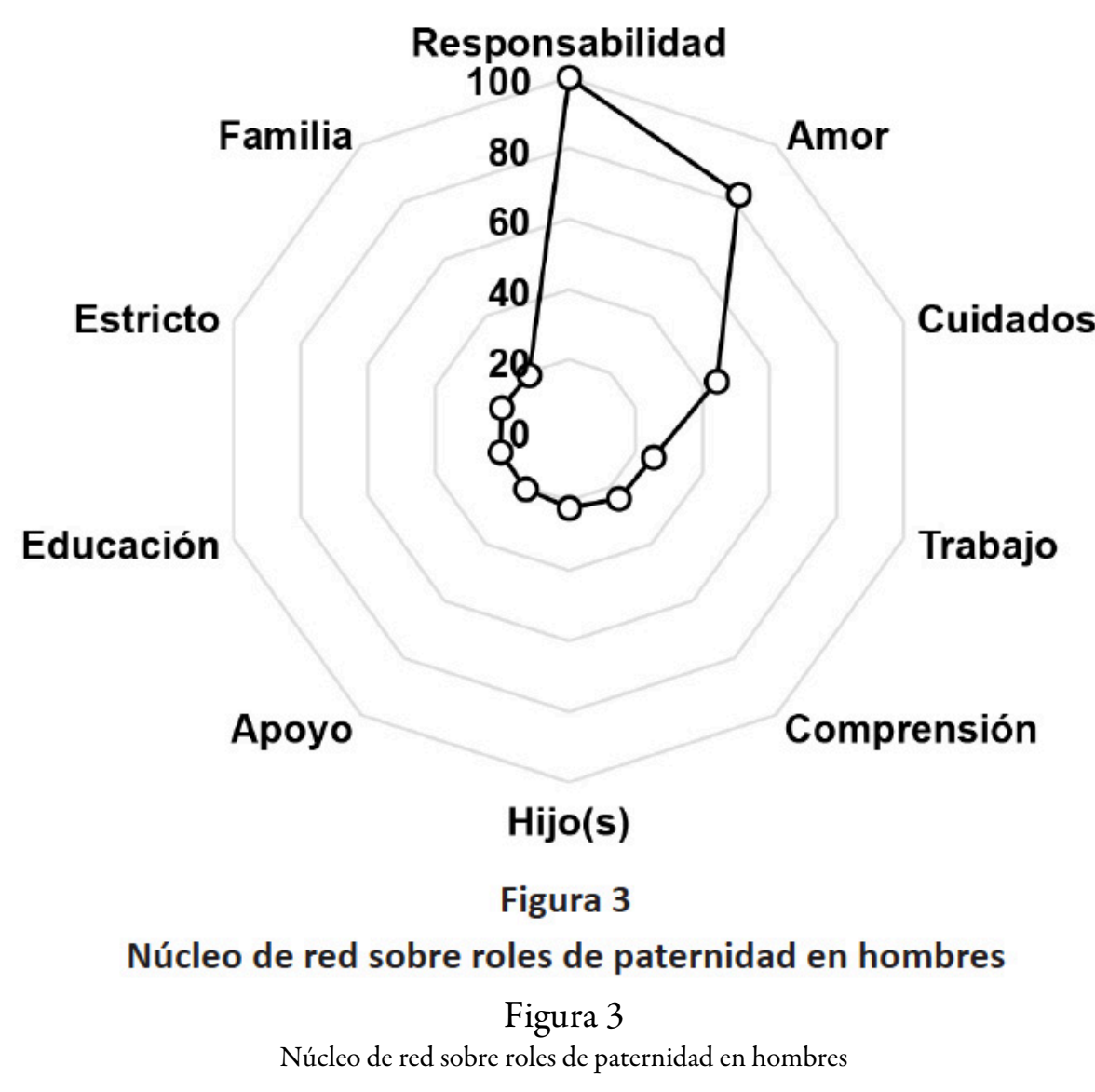




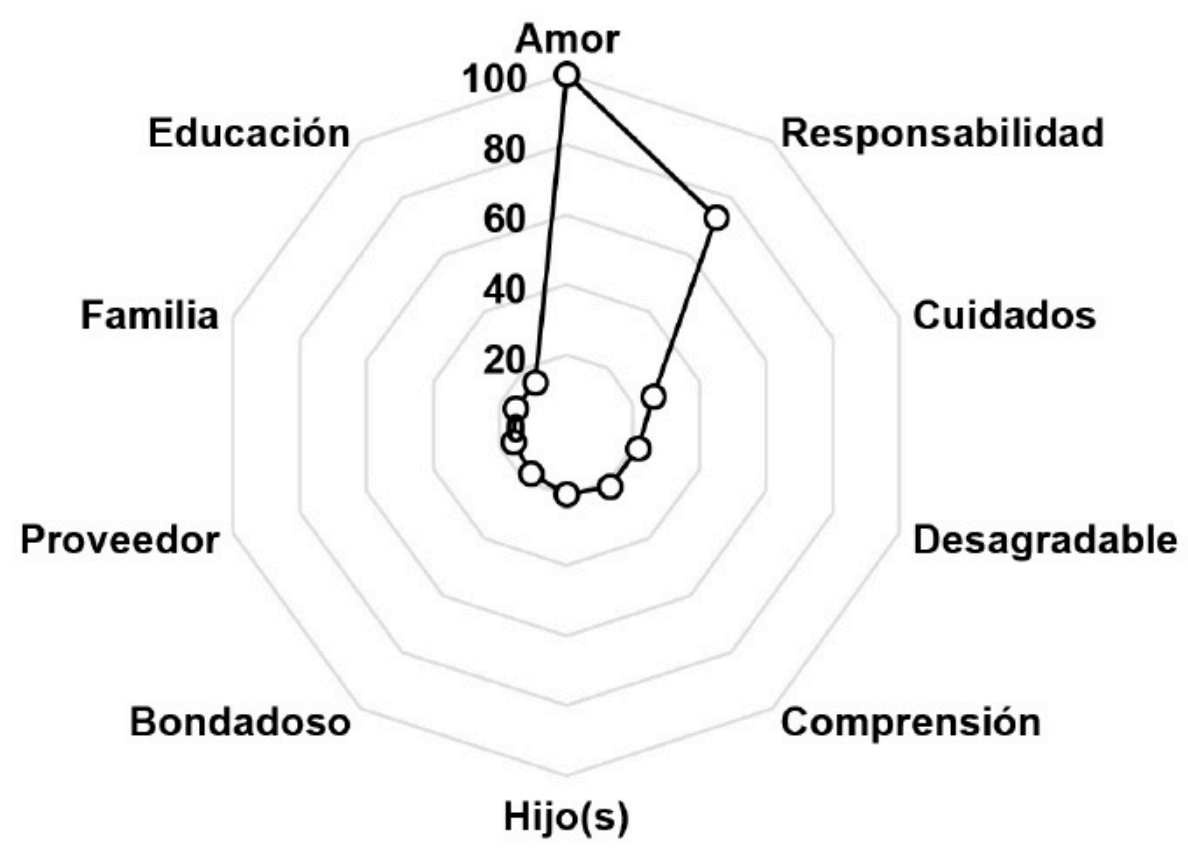

Figura 4

Núcleo de red sobre roles de paternidad en mujeres

Figura 4

Núcleo de red sobre roles de paternidad en mujeres

De igual modo, como se muestra en la Tabla 2, encontramos diferencias relevantes entre los pesos semánticos entre sexos, así como entre las distancias semánticas cuantitativas de cada estímulo intrasexo.

Expectativas Relacionadas con la Paternidad

Como se mencionó con anterioridad, este rubro se encuentra compuesto por dos estímulos, uno enfocado en el futuro y el otro, en el presente. Respecto al primero ("Mi pareja esperaría que, como padre, yo...” / "Espero que mi pareja como padre"), se encontró un tamaño de red más grande en mujeres con 110 palabras emitidas, en contraste con las 87 palabras referidas por los hombres. Los núcleos de red presentan un índice de consenso grupal de 50\%, con el cual observamos que las expectativas futuras hacia la paternidad, para ambos, se concentran en que sea responsable, amoroso, cuidadoso, que brinde apoyo y atención; sin embargo, difieren en características como respetuoso, comprensivo, felicidad, bondad y que eduque, en el caso de las mujeres; y que trabaje, sostenga, cumpla, sea comprometido y dinero, en cuanto a hombres (Ver Figura 5 y 6 ). 
Tabla 2

\begin{tabular}{|c|c|c|c|c|c|}
\hline \multicolumn{6}{|c|}{ Estímulo: Mi pareja como padre / Yo como padre } \\
\hline \multicolumn{3}{|c|}{ Mujeres } & \multicolumn{3}{|c|}{ Hombres } \\
\hline Núcleo de red & ps & dsc & Núcleo de red & ps & dsc \\
\hline Amor & 327 & 100 & Responsabilidad & 240 & 100 \\
\hline Responsabilidad & 239 & 73.08 & Amor & 198 & 82.5 \\
\hline Cuidados & 86 & 26.29 & Cuidados & 106 & 44.16 \\
\hline Desagradable & 71 & 21.71 & Trabajo & 61 & 25.41 \\
\hline Comprensión & 70 & 21.4 & Comprensión & 58 & 24.16 \\
\hline Hijo(s) & 65 & 19.87 & $\mathrm{Hijo}(\mathrm{s})$ & 53 & 22.08 \\
\hline Bondadoso & 56 & 17.12 & Apoyo & 50 & 20.83 \\
\hline Proveedor & 52 & 15.9 & Educación & 49 & 20.41 \\
\hline Familia & 50 & 15.29 & Estricto & 48 & 20 \\
\hline Educación & 50 & 15.29 & Familia & 38 & 19.19 \\
\hline
\end{tabular}

Tabla 2

Núcleo de red, peso semántico y distancia semántica cuantitativa de los roles paternidad en hombres y mujeres

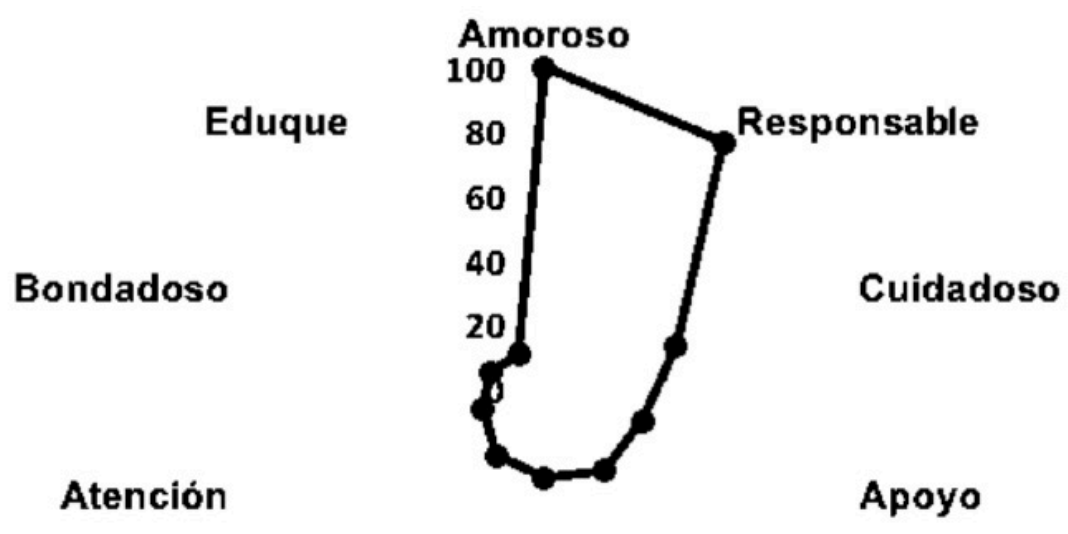

Felicidad

Respetuoso

\section{Comprensivo}

Figura 5

Núcleo de red de expectativas a futuro de paternidad en mujeres

Figura 5

Núcleo de red de expectativas a futuro de paternidad en mujeres 


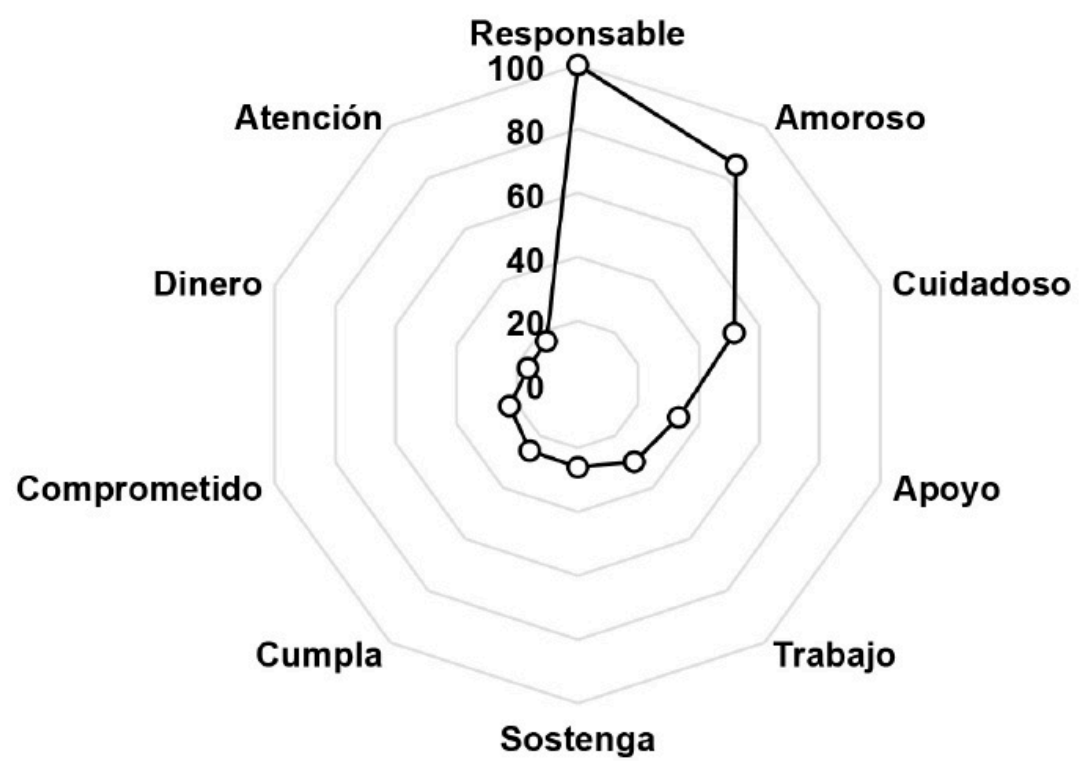

Figura 6

Núcleo de red de expectativas a futuro de paternidad en hombres

Figura 6

Núcleo de red de expectativas a futuro de paternidad en hombres

Como se muestra en la Tabla 3, los pesos semánticos entre sexos difieren a pesar de las semejanzas existentes entre las definidoras; no obstante, en cuanto a la distancia semánticas cuantitativa se identifica cierta fluidez entre las definidoras de ambos sexos.

\section{DISCUSIÓN}

En términos generales, la paternidad continúa asociándose a características fuertemente culturales como la autoridad, responsabilidad y provisión (Castillo, 2015; Correa et al., 2013; Nieri, 2012), aunque en menor escala a la esperada. A su vez, se vislumbran indicadores descriptivos relacionados con componentes afectivos, de cuidado y apoyo, que dan evidencia, como indica Bonino (2003), de los cambios ideológicos que han permeado el escenario de la paternidad tanto en hombres como en mujeres, ya perceptibles como creencia, aunque aún no como norma social, tal y como señala el estudio de García (2011).

En particular, respecto al significado psicológico, se observa la correspondencia de la paternidad hacia indicadores clave como el ser hombre, tener hijos y cumplir ciertas características como la responsabilidad y provisión, asociados directamente con las expectativas sociales del rol (Saldívar et al., 2015), así como afectivas. A su vez, se encuentran similitudes en los indicadores afectivos y de cuidado que podrían precisar aceptación o expectativa hacia prácticas más activas afectivamente sobre las de provisión económica, en la cual anida la posibilidad de pluralizar, reconocer y validar las diferentes formas de ser padre en la actualidad. 
En cuestión de roles, ambos sexos brindan mayor valor a los relacionados con responsabilidad y cariño, por encima de otros como el trabajo y la educación. A su vez, se encuentra que, tanto en hombres como en mujeres, los cuidados son un concepto central. Estos resultados contrastan con lo encontrado en la literatura, donde se brinda mayor importancia al rol de proveedor (Bonino, 2003; Castillo, 2015; Correa et al., 2013). No obstante, los varones mencionan como componente importante el trabajo, lo que posiblemente responde a la relación directa entre trabajo, dinero y provisión, así como, al hecho de representar al género al cual se le resalta más vívidamente dicha expectativa.

De igual modo, se destaca la comprensión como un elemento sustancial, al igual que la educación, esta última también relacionada con las funciones esperadas de los padres (Silverstein et al., 1999, citado en Paterna et al., 2005). Cabe destacar la posible relación del rol paterno con la familia, lo que remite al carácter relacional o bidireccional de la paternidad con la pareja y los hijos (Bonino, 2003; Torres, Salguero, \& Ortega, 2005).

Tabla 3

\begin{tabular}{|c|c|c|c|c|c|}
\hline \multicolumn{6}{|c|}{ Estímulo: Mi pareja como padre / Yo como padre } \\
\hline \multicolumn{3}{|c|}{ Mujeres } & \multicolumn{3}{|c|}{ Hombres } \\
\hline Júcleo de red & ps & dsc & Núcleo de red & ps & dsc \\
\hline Amoroso & 347 & 100 & Responsable & 315 & 100 \\
\hline Responsable & 329 & 94.81 & Amoroso & 267 & 84.76 \\
\hline Cuidadoso & 150 & 43.22 & Cuidadoso & 163 & 51.74 \\
\hline Apoyo & 112 & 32.27 & Apoyo & 105 & 33.33 \\
\hline Respetuoso & 109 & 31.41 & Trabajo & 95 & 30.15 \\
\hline Comprensivo & 96 & 27.66 & Sostenga & 82 & 26.03 \\
\hline Felicidad & 88 & 25.36 & Cumpla & 81 & 25.71 \\
\hline Atención & 69 & 19.88 & Comprometido & 71 & 22.53 \\
\hline Bondadoso & 61 & 17.57 & Dinero & 52 & 16.5 \\
\hline Educación & 46 & 13.25 & Atención & 52 & 16.5 \\
\hline
\end{tabular}

Tabla 3

Núcleo de red, peso semántico y distancia semántica cuantitativa de las expectativas futuras de paternidad en hombres y mujeres

Es importante señalar la aparición del descriptivo desagradable presente en las mujeres, el cual no aparece dentro de las definidoras enlistadas por los hombres. Sería relevante clarificar en un estudio posterior, las acciones, creencias o sentimientos que perciben en sus parejas dentro de este rol que las lleva a concebirlos de esta manera.

Respecto a las expectativas de la paternidad, coincide con los roles afectivos, de responsabilidad, cuidado y atención, aunque los hombres se adjudican un peso más hacia lo económico y las mujeres hacia lo afectivo, posiblemente debido a que, como indica García, Salguero y Pérez (2010) y Saldívar et al. (2015), se encuentran fuertemente ligados a estereotipos de género socializados desde la infancia.

Asimismo, los resultados anteriores coinciden con lo expuesto por Bonino (2003), donde señala la existencia de la expectativa social hacia el padre de responsabilidad y provisión, así como la posibilidad de 
estar presente o no en el ambiente familiar, siempre y cuando, cumpla con ser responsable. Cabe destacar que dicha expectativa se encontró matizada por indicadores afectivos, sobre todo en las mujeres, velando por un rol más andrógino y expresivo emocionalmente del padre. Esto demuestra la transición cultural en la dicotomía genérica, donde las mujeres presentan un mayor avance social (García-Campos, 2008). No obstante, el cuestionamiento de la experiencia masculina en cuanto a las disidencias de su ejercicio paterno queda en el tintero.

De igual modo, sería importante dilucidar sobre a lo que se refieren los jóvenes cuando hablan de responsabilidad, ya que en sus apariciones suelen ligarse con definidoras referentes a la esfera económica, afectiva y de crianza. La paternidad responsable comprende estos tres ámbitos, y sería necesario esclarecer si su concepción de responsabilidad los integra a los tres o prioriza alguno(s) sobre otro(s).

Se destaca la bidireccional de dichos constructos entre hombres y mujeres, donde ambos han presentado indicadores hacia una dinámica cultural direccionada hacia un ejercicio disidente de la paternidad. No obstante, resulta interesante que en los estímulos donde los hombres dan más énfasis a los cuidados y afecto, las mujeres lo hacen hacia la provisión, y viceversa. Lo que lleva a reflexionar sobre la posibilidad de acceder a dichas prácticas genéricas y a la influencia de la pareja en esta transición, ¿bastará con sólo presentar el interés? Se espera que los varones cambien su actuar paterno y masculino, aunque ¿la sociedad estará preparada para recibir culturalmente dicho cambio?

Por otra parte, los resultados de esta investigación permiten constatar la vigencia de algunas expectativas respecto a los roles tradicionales de género, aunque sin duda ya se vislumbra un cuestionamiento a dichos marcajes; no obstante, la tarea de las nuevas generaciones será deconstruir el concepto de su paternidad y las expectativas en pareja que se tienen de ésta. Por lo tanto, promover un encuadre teórico que matice la diversidad de los roles e identidades en los individuos se vuelve una tarea importante para, así, trascender de la categorización que suele marcar nuevas formas de valorar a los hombres, las mujeres y, por ende, las acciones, pensamientos y relaciones que tienen entre sí. Los alcances de esta investigación versan en reconocer la importancia de seguir estudiando este concepto en poblaciones jóvenes que permitan ampliar la visión de la paternidad hacia otros escenarios como los médicos, institucionales y laborales. Además abre camino hacia sustentar intervenciones sobre la salud sexual y reproductiva dentro de este grupo poblacional para, así, dar paso al trabajo con los mismos en un proyecto de vida en el que se concientice sobre las implicaciones de ejercer sus derechos humanos, en pro de establecer relaciones padres e hijas e hijos más saludables en las familias. Finalmente se sugieren técnicas de naturaleza cuantitativa y cualitativa a muestras más amplias y diversas, con la finalidad de rescatar y enriquecer la diversidad de este fenónemo en los diferentes escenarios socioculturales. 


\section{REFERENCIAS}

Aguirre, R., \& Guell, P. (2002). Hacerse hombres. La construcción de la masculinidad en los adolescentes y sus riesgos. Chile: Organización Panamericana de la Salud.

Barker, G., Aguayo, F., Kimelman, E., \& Figueroa, J. (2011). Los hombres en las políticas de género. En G. Barker \& F. Aguayo (Eds.), Masculinidades y politicas de equidad de género: Reflexiones a partir de la encuesta. Images y una revisión de politicas en Brasil, Chile y México (pp. 58-85). Río de Janeiro, Brasil: Promundo.

Barker, G., Aguayo, F., Correa, P., Contreras, J. M., Segundo, M., Nascimiento, M., \& Figueroa, J. G. (2011). Encuesta images. Datos comparados de Brasil, Chile y México. En G. Barker \& F. Aguayo (Eds.), Masculinidades y politicas de equidad de género: Reflexiones a partir de la encuesta. Images y una revisión de politicas en Brasil, Chile y México (pp. 14- 57). Río de Janeiro, Brasil: Promundo.

Bonino, L. (2003). Las nuevas paternidades. Cuadernos de Trabajo Social, 16(1). $171-182$.

Brañas, P. (1997). Atención del pediatra al adolescente. Pediatría Integral, 2, 207-216.

Castillo, A. (2015). La práctica social de la maternidad y de la paternidad en jóvenes estudiantes de nivel superior: Un acercamiento a la problemáticas cotidianas enfrentadas durante la vida académica. Estudios sobre las Culturas Contemporáneas, 21(2), 103-123.

Comisión Económica para América Latina y el Caribe [Cepal] (2001). Diagnóstico sobre paternidad responsable y propuestas para un programa nacional en el Salvador. México: Cepal.

Connell, R. W. (2003). Adolescencia en la construcción de masculinidades contemporáneas. En J. Olavarría (Ed.),Varones adolescentes: Género, identidades y sexualidades en América Latina (pp. 53-67). Santiago, Chile: Flacso-Chile.

Correa, F., García, L., \& Saldívar, A. (2013). Estereotipo de paternidad e identidad de género en adolescentes de la Ciudad de México. Revista Iberoamericana de Psicologia: Ciencia y Tecnologia, 6(1), 41-50.

Cubillas, M., Valdez, E., Domínguez, S., Román, R., Hernández, A., \& Zapata, J. (2016). Creencias sobre estereotipos de género de jóvenes universitarios del norte de México. Diversitas: Perspectivas en Psicología, 12(2), 217-230.

Cultura Salud/ Sename (2011). Previniendo la violencia con jóvenes. Talleres con enfoque de género y masculinidades. Manual para facilitadores y facilitadoras. Santiago, Chile: Servicio Nacional de Menores / CulturaSalud / eme.

Díaz-Guerrero, R., \& Castillo-Vales, V. M. (1981). El enfoque culturacontracultura y el desarrollo cognitivo y de la personalidad en escolares yucatecos. Enseñanza e Investigación en Psicología, 7(1), 5-26.

Díaz-Loving, R., Rivera, S., Villanueva, G., \& Cruz, L. (2011). Las premisas histórico-socioculturales de la familia mexicana: Su exploración desde las creencias y las normas. Revista Mexicana de Investigación en Psicología, 3(2), 128-142.

Díaz-Loving, R., Saldívar, A., Armenta-Hurtarte, C., Reyes, N., López, F., Moreno, M., Romero, A., Hernández, J., Domínguez, M., Cruz, C., \& 
Correa, F. (2015). Creencias y normas en México: Una actualización del estudio de las premisas psico-socio-culturales. Psykhe, 24(2), 1-25. http:// dx.doi.org/10.7764/psykhe.24.2.880

Figueroa, J., \& Flores, N. (2012). Prácticas de cuidado y modelos emergentes en las relaciones de género. La experiencia de algunos varones en México. La ventana. Revista de Estudios de Género, 4(35), 7-57.

Figueroa, J., González, E., \& Solís, V. (1981). Una aproximación al problema del significado: Las redes semánticas. Revista Latinoamericana de Psicología, 13(3), 447-468.

Gaete, V. (2015). Desarrollo psicosocial del adolescente. Revista Chilena de Pediatria, 86(6), 436-443. https://doi.org/10.1016/j.rchipe.2015.07.005

García, E., Salguero, A., \& Pérez, G. (2010). Expectativas y estereotipos de género en la relación entre padres e hijas. Enseñanza e Investigación en Psicología, 15(2), 325-341.

García-Campos, T. (2008). Cultura tradicional y masculinidad feminidad. Interamerican Journal of Psychology, 42(1), 58-68.

García, L. (2011). El impacto de la cultura en los significados de las premisas histórico-socio-culturales. Revista Mexicana de Investigación en Psicología, 3(2), 154-158.

Mena, P., \& Torres, L. (2013). Prácticas paternas en divor- ciados, viudos y abandonados. En J. C. Ramírez \& J. Cervantes. (Eds.), Los hombres en México. Veredas recorridas y por andar. Una mirada a los estudios de género de los hombres, las masculinidades (pp. 71-90). Guadalajara, México: Universidad de Guadalajara.

Minuchin, S., \& Fishman, H. (1984). Técnicas de terapia familiar. Barcelona, España: Paidós.

Nieri, L. (2012). Paternidad y maternidad: Aproximaciones psicológicas y socioculturales. Poiesis, Revista Electrónica de Psicología Social, 23, 1-12. h ttps://doi.org/10.21501/issn.1692-0945

Paterna, C., Martínez, C., \& Rodes, J. (2005). Creencias de los hombres sobre lo que significa ser padre. Interamerican Journal of Psychology, 39(2), 275-284.

Ramírez, J. (2006). ¿Y eso de la masculinidad? Apuntes para una discusión. En G. Careaga \& S. Cruz (Eds.), Debates sobres masculinidades. Poder, desarrollo, politicas públicas y ciudadania (pp. 31-56). México: Universidad Nacional Autónoma de México.

Reyes-Lagunes, I. (1993). La redes semánticas naturales, su conceptualización y su utilización en la construcción de instrumentos. Revista de Psicología Social y Personalidad, 9(1), 81-97.

Saldívar, A., Díaz-Loving, R., Reyes, N., Armenta, C., López, F., Moreno, M., Romero, A., Hernández, J., \& Domínguez, M. (2015). Roles de género y diversidad. Validación de una escala en varios contextos culturales. Acta de Investigación Psicológica, 5(3), 2124-2148. https://doi.org/10.1016/S200 7-4719(16)30005-9

Salguero, A. (2006). Identidad, responsabilidad familiar y ejercicio de la paternidad en varones del Estado de México. Papeles de Población, 12(48), 155-179. 
Torres, L., Salguero, A., \& Ortega, P. (2005). Efectos de la presencia de los varones en el desarrollo psicológico infantil. Psicología y Salud, 15(1), 113-120. 